Revista de Psicología Vol. 35 (2), 2017 (ISSN 0254-9247)

\title{
Estilos y estrategias de aprendizaje en estudiantes universitarios de Buenos Aires
}

\author{
Agustín Freiberg Hoffman ${ }^{1}$, Ruben Ledesma ${ }^{2}$, Mercedes Fernández Liporace ${ }^{3}$ \\ Universidad de Buenos Aires ${ }^{1,3}$, Universidad Nacional de Mar del Plata², \\ Consejo Nacional de Investigaciones Cientificas y Técnicas (CONICET) $)^{1,2,3}$
}

Los estilos y las estrategias de aprendizaje describen las preferencias de los estudiantes durante las actividades de aprendizaje que involucran el incorporar y conciliar exitosamente información novedosa con la preexistente. Los participantes fueron 438 estudiantes universitarios de diferentes carreras, entre 19 y 36 ańos. Los resultados evidencian diferencias significativas en algunas dimensiones de estilos y estrategias según variables sociodemográficas y académicas, así como asociaciones significativas entre algunas estrategias y la cantidad de recursos tecnológicos que emplean los alumnos. Se verificó la influencia de determinadas estrategias y estilos sobre el rendimiento académico. Estilos y estrategias varían en los estudiantes según características académicas y sociodemográficas. Se han aislado algunos estilos y estrategias que afectan significativamente el rendimiento de todos los educandos analizados.

Palabras clave: estilos de aprendizaje, estrategias de aprendizaje, estudiantes universitarios, rendimiento académico, metacognición.

\section{Learning Styles and Learning Strategies in College Students from Buenos Aires}

Learning styles and learning strategies describe students' preferences while they are developing learning activities— grasping and transforming experience—, successfully integrating new and previous information-. 438 college students between 19 and 36 years old participated. Significant differences by sociodemographic and academic variables were verified both in styles and strategies in several dimensions, as well as significant associations between some strategies and the number of technological resources employed by students. Findings

1 Doctor en Psicología. Becario posdoctoral y profesor en la Universidad de Buenos Aires. Dirección postal: Vallejos $23794^{\circ}$ Piso (1419) Capital Federal. Argentina. Contacto:afreiberg@psi. uba.ar / agustinfreiberg@gmail.com

2 Doctor en Psicología. Profesor e Investigador en la Universidad Nacional de Mar de Plata. Dirección postal: Río Negro 3922 (7600). Mar del Plata. Argentina. Contacto: rdledesma@ gmail.com

3 Doctora en Psicología. Profesora e investigadora en la Universidad de Buenos Aires. Dirección postal: General Juan Lavalle 2353 (1052). Capital Federal. Argentina. Contacto: mliporac@ psi.uba.ar

https://doi.org/10.18800/psico.201702.006 
report the influence of specific strategies and styles on academic achievement, whereas styles and strategies vary in students according to academic and sociodemographic features. However certain styles and strategies significantly influencing academic achievement in students composing the whole sample were identified.

Keywords: learning styles, learning strategies, college students, academic achievement, metacognition.

\section{Estilos e Estratégias de Aprendizagem em Estudantes Universitários de Buenos Aires}

Os estilos e as estratégias de aprendizagem descrevem as preferências dos estudantes em atividades de aprendizagem -incorporar e conciliar com sucesso informação nova com a preexistente- - Participaram na investigação 438 estudantes universitários de diferentes carreiras, com idades entre 19 e 36 anos. Acharam-se diferenças significativas em algumas dimensōes de estilo e estratégias segundo variáveis sociodemográficas e académicas, tanto como associaçóes significativas entre algumas estratégicas e a quantidade de recursos tecnológicos empregados pelos alunos. Checou-se a influência de determinadas estratégias e estilos sobre o rendimento académico. Estilos e estratégias variam nos estudantes conforme as características académicas e sociodemográficas. Foram isolados alguns estilos e estratégicas que afetam significativamente o rendimento dos alunos analisados.

Palavras-chave: estilos de aprendizagem, estratégicas de aprendizagem, estudantes universitários, rendimento académico, metacognição. 
Estilos y estrategias de aprendizaje en estudiantes universitarios de Buenos Aires / Freiberg et al.

Conocer las preferencias de los estudiantes universitarios a la hora de aprender se ha vuelto en la última década un tema de gran interés (AlKhasawneh, 2013; Hallin, 2014; Williams, Brown \& Etherington, 2013). Esta información posibilita a los diferentes actores del medio educativo superior adoptar medidas tendientes a facilitar en los alumnos la incorporación de información novedosa y su articulación con el conocimiento previo (Ponce de León Castañeda, Varela-Ruiz, LozanoSánchez, Ortiz-Montalvo \& Ponce-Rosas, 2010; Ventura, Moscoloni \& Gagliardi, 2012). De esta manera, el cuerpo docente podría modificar sus prácticas pedagógicas, empleando recursos ajustados a los perfiles estudiantiles. Las instituciones, por su parte, se encontrarían en condiciones de mejorar sus planes de estudio a fin de reforzar la articulación entre contenidos teóricos y prácticos, facilitando así la aprehensión de conocimientos. Por su parte, los estudiantes podrían, en virtud de lo anterior, adaptar sus preferencias para aprender a las características de los perfiles descriptos para la carrera por ellos elegida mediante el entrenamiento de diferentes habilidades cognitivas y metacognitivas bajo la guía de profesionales psicólogos del ámbito educativo. Todo esto facilitaría la incorporación de la información disciplinar de modo más fluido y, a la vez, orientaría más eficazmente las elecciones de especialidades realizadas por el estudiantado.

En concordancia con lo anterior, las preferencias para aprender se encuentran asociadas a las nociones de estilos y estrategias de aprendizaje, siendo ambos conceptos fundamentales para el estudio de los procesos de aprendizaje (Gravini \& Iriarte, 2008; Ventura, 2011).

\section{Estilos y estrategias de aprendizaje}

Los estilos de aprendizaje se definen como un conjunto de características intelectuales y de personalidad que configuran el modo en que los estudiantes perciben, interactúan y responden frente a situaciones 
de aprendizaje (Curry, 1983; Keefe, 1988). El análisis de este constructo permite responder a la pregunta acerca de por qué dos personas que enfrentan una misma situación de aprendizaje logran asimilar distinta cantidad de información (Honey \& Mumford, 1986). Si bien los estilos de aprendizaje se hacen presentes en todos los individuos, no se encuentran necesariamente desarrollados de modo parejo. El predominio de un estilo sobre otros facilita el aprendizaje de un tipo de información específica. Así, la asimilación de cierta clase de conocimientos se vería favorecida por la posesión de una estructura de estilos acorde a la situación de aprendizaje (Kolb \& Kolb, 2005). Los contenidos, metodologías y exigencias varían de una carrera a otra. Por eso la incorporación de conocimientos por parte del alumno se vuelve más sencilla a medida que su configuración de estilos se asemeja al requerido por la disciplina que desea cursar.

Con la intención de mejorar los aprendizajes se han investigado las preferencias estudiantiles en diferentes carreras, a fin de facilitar información útil que permita a los profesores adaptar sus estilos de enseñanza a tales características (Aragón-García \& Jiménez-Galán, 2009; Caballero-Vargas, 2015). Por otra parte, aunque con mayor dificultad, los estudiantes pueden también adecuar paulatinamente sus estilos a las demandas de la carrera por ellos elegida, modificando sus preferencias para aprender a medida que avanzan en sus trayectos académicos (Bahamón, Vianchá, Alarcón \& Bohórquez, 2012). Quienes no reconfiguren sus estilos a pesar de necesitarlo, probablemente presentarán mayores dificultades para asimilar los contenidos, insumiéndoles ello mayor tiempo y esfuerzo. Esto último suele llevar, en ocasiones, al fracaso universitario y la posterior deserción del sistema (Biggio, Vázquez \& García, 2015; Borracci \& Arribalzaga, 2015).

Si bien existen diversos modelos que permiten comprender el fenómeno de los estilos de aprendizaje, uno de los más difundidos corresponde a Kolb (1979). Este autor plantea que la generación de conocimiento se encuentra determinada por la transformación de la experiencia percibida y que este proceso tiene lugar a partir de dos componentes, uno perceptivo y otro de procesamiento. El primero se rela- 
ciona con la captación y conceptualización de la experiencia y el segundo con el pensamiento y la comprobación de la información novedosa. A la vez, cada dimensión se hipotetiza integrada por dos procesos dialécticos que participan en toda situación de aprendizaje, correspondiéndole a la perceptual los de Experiencia Concreta (EC) y Conceptualización Abstracta (CA) y a la procesual, la Observación Reflexiva (OR) y la Experimentación Activa (EA). Estas cuatro operaciones configuran en los estudiantes sus preferencias para percibir y procesar la información, derivándose de su combinación los estilos formulados en el modelo (Kolb, 1984). Enuncia de este modo los estilos Adaptador (EC-EA), Asimilador (CA-OR), Convergente (CA-EA), y Divergente (EC-OR). Así, los adaptadores son adeptos a desafíos novedosos, disfrutan de experiencias prescindiendo del análisis lógico, guían sus acciones mediante sus intuiciones y sentimientos, y pueden delegar los aspectos técnicos en los otros ya que confían en criterios ajenos. Los asimiladores se destacan por manejar y comprender una amplia variedad de información, son poco sociables, disfrutan del análisis de conceptos abstractos buscando la coherencia y validez lógica de las ideas. Los convergentes, por su parte, son poco permeables a recomendaciones ajenas, disfrutan de la puesta a prueba de modelos novedosos, simulaciones y ensayos en laboratorios. Esto les hace preferir trabajar solos, resolviendo problemas técnicos y evitando toda actividad social que implique alguna clase de relación interpersonal. Finalmente, los divergentes, se distinguen por su habilidad para observar las situaciones desde múltiples puntos de vista y generar ideas novedosas. Son personas con amplios intereses culturales a las que les gusta recopilar toda clase de información. El modelo propuesto por Kolb (1984) sienta la base a partir de la cual parten otros desarrollos teóricos, como el de Alonso, Gallego y Honey (1994), quienes reducen los estilos Adaptador, Asimilador, Divergente y Convergente a sus cuatro procesos subyacentes - Experiencia Concreta, Observación Reflexiva, Conceptualización Abstracta, Experimentación Activa-, renombrándolos como estilos Activo, Reflexivo, Teórico y Pragmático. Definen así a los activos como aquellos sujetos que gustan de participar en experiencias novedosas, poseyendo una amplia apertura 
mental y un elevado interés por resolver problemáticas actuales. Los reflexivos por su lado, se centran en la recolección y análisis de información diversa proveniente de múltiples fuentes. Son individuos preocupados por abarcar todas las aristas posibles de una problemática a fin de contar con un cúmulo de información que les permita arribar a conclusiones lo más robustas posibles. Por su parte, los teóricos, se distinguen por el grado de complejidad de las teorías que generan a partir de la integración de un conjunto de observaciones y hechos. Se trata de personas racionales que intentan ser lo más objetivas posibles y con una fuerte tendencia al perfeccionismo. En último lugar se tienen a los pragmáticos que son individuos que gustan de poner en práctica las ideas novedosas, descubriendo el aspecto positivo de cada una de ellas. Suelen ser impacientes y rápidamente actúan cuando una idea les atrae, buscando soluciones concretas a los problemas. Tanto el modelo de Kolb (1984), como el de Alonso et al (1994) se emplean con frecuencia en diversas investigaciones realizadas en países de habla hispana entre los que se incluye Argentina (e.g. Maureira et al., 2015; Sánchez-Socarrás, Donat-Roca, Fornons-Fontdevilla \& Vaqué-Crusellas, 2015; Villalba, 2015; Zapata-Castañeda et al., 2012).

Pasando ahora al constructo estrategias de aprendizaje, ellas designan las técnicas que se emplean en la resolución de tareas específicas a la hora de aprender. Se definen como actividades que el estudiante realiza durante la situación de aprendizaje con el propósito de influir sobre los procesos cognoscitivos y afectivos, permitiéndole alcanzar un determinado fin (Weinstein \& Mayer, 1986). Se diferencian tres grandes categorías que agrupan las diferentes estrategias de aprendizaje: cognitivas, metacognitivas y afectivas. Las cognitivas remiten a técnicas para aprender, codificar, comprender y recordar la información en función de determinadas metas de aprendizaje. Las metacognitivas se vinculan con la planificación, control y evaluación que hacen los estudiantes de sus procesos mentales. Las afectivas se relacionan con los motivos, intenciones, metas y emociones que intervienen en la asimilación de conocimientos (Escurra-Mayaute, 2006; Valle, González, Cuevas \& Fernández, 1998). En la enseñanza universitaria 
las estrategias de aprendizaje juegan un papel fundamental. Su carácter intencional permite trazar un plan de acción de modo tal que, a partir de la información que se desea incorporar, sea posible seleccionar aquellas conductas que faciliten la tarea. De este modo es posible mejorar el quehacer académico a través de tres actividades educativas, que son la prevención -identificar y reemplazar estrategias poco eficaces por otras de mayor eficacia-, optimización -estimular el empleo de estrategias eficaces que el estudiante pone en juego en el presente- y recuperación - localizar estrategias responsables del bajo rendimiento e intentar mejorarlas- (Beltrán, 2003).

Llegado a este punto es necesario destacar la diferencia entre estilos y estrategias de aprendizaje. Mientras los primeros remiten a lo que hace habitualmente un sujeto para incorporar y conciliar información novedosa con la preexistente, las segundas, organizadas y controladas por los estilos, refieren a acciones, técnicas u operaciones que la persona emplea para resolver del modo más sencillo posible una tarea particular (Biggs, 1988). Así, se trataría de dos caras de una misma moneda, centrándose la primera en elementos inherentes a la persona -aspectos cognitivos y de personalidad- examinando el patrón de conductas que comúnmente cada uno pone en juego, independientemente de la situación de aprendizaje. Las estrategias, en cambio, se refieren a técnicas y procedimientos que debieran activarse para resolver apropiadamente una tarea específica (Navarro-Jiménez, 2008).

El análisis conjunto de los estilos y las estrategias de aprendizaje apunta a obtener información que, por un lado posibilite a los profesores adaptar sus modalidades de enseñanza a los estilos estudiantiles $y$, por otro permita a los educandos desarrollar estrategias que faciliten la incorporación de contenidos académicos específicos (Bahamón, Vianchá, Alarcón \& Bohórquez, 2013; Mehrdad \& Ahghar, 2012).

\section{Estilos y estrategias de aprendizaje en estudiantes universitarios}

Estilos y estrategias de aprendizaje han sido constructos ampliamente investigados en el ámbito universitario. Por un lado, se los ha estudiado en relación a variables sociodemográficas tales como sexo, 
edad, nivel educativo de los padres, entre otras (Aguilar, 2010; Cuña, Gutiérrez, Barón \& Labajos, 2014; Martín-García \& RodríguezConde, 2003). De este modo se intenta ubicar aquellas características sociodemográficas que podrían contribuir al desarrollo de algún estilo por sobre otros o bien al empleo de determinada clase de estrategias.

Por otra parte sendos constructos han sido también examinados en relación a otros conceptos psicológicos como son los estilos de pensamiento, actitudes, personalidad, y motivación, entre otros (Allueva-Torres \& Bueno-García, 2011; Donche, De Maeyer, Coertjens, Van Daal \& Van Petegem, 2013; Flores-Mejía, Velázquez-Gatica \& Gaona-Rojas, 2014). Tales trabajos informan sobre aquellas variables que tendrían un papel determinante en la configuración de los estilos y estrategias, ayudando a la planificación de actividades centradas en el entrenamiento de sus distintas dimensiones.

El reciente desarrollo tecnológico ha llevado a varios investigadores a estudiar la relación entre el uso de la tecnología, en tanto herramienta facilitadora de los aprendizajes, y los diferentes estilos y estrategias de aprendizaje (Calvache-Sánchez, Gómez-Ramírez \& Poveda-Alonso, 2013; Fernández-Zalazar \& Neri, 2013; Moya-Martínez, HernándezBravo, Hernández-Bravo \& Cózar-Gutiérrez, 2011; Pujol, 2008). Estos trabajos se centran en conocer aquellas cualidades personales facilitadoras del uso de tales recursos.

Estilos y estrategias son también abordados en función de distintas características académicas, tales como el rendimiento, la modalidad de cursado de clases — presencial, e-learning, b-learning-, carrera, avance en el ciclo académico, tipo de educación secundaria - bachiller o técnico-, entre otras (Blumen, Rivero \& Guerrero, 2011; Broadbent \& Poon, 2015; Camacho, Chiappe-Laverde \& López de Mesa, 2012; Chen-Kang \& Manjit-Singh, 2015; Loret de Mora Garay, 2011; Muñetón, Pinzón, Alarcón \& Olaya, 2013; Ortiz-Fernández et al., 2014; Ortiz-Ojeda \& Canto-Herrera, 2013; Padilla-Miranda, Gutiérrez-Fonseca \& Cabrera-Campos, 2013; Peixoto, Peixoto \& Alves, 2012; Simsec \& Balaban, 2010). Los trabajos orientados en esta dirección dan cuenta del modo diferencial en que los estilos y estrategias se presentan 
en estudiantes con distintas particularidades académicas. Entre las variables académicas existe un especial énfasis puesto en el análisis de los estilos y las estrategias de aprendizaje en relación al rendimiento estudiantil (e.g. Abidin, Rezaee, Abdullah \& Singh, 2011; Almigbal, 2015; Jiraporncharoen, Angkurawaranon, Chockjamsai, Deesomchok \& Euathrongchit, 2015; Muelas \& Navarro, 2015; Yip, 2012). Estas investigaciones buscan aislar aquellas dimensiones relacionadas con el desempeño estudiantil a fin de construir perfiles educativos. Plantean, además, a partir de esta información, diseñar acciones concretas tendientes a evitar el fracaso y la deserción académica.

Dada la relevancia que los estilos y las estrategias han mostrado poseer dentro del ámbito universitario es que la presente investigación se plantea los siguientes interrogantes: 1) ¿Las variables sociodemográficas introducen diferencias estadísticamente significativas en los estilos y estrategias de aprendizaje de los alumnos? 2) ¿El uso de recursos tecnológicos se relaciona significativamente con el empleo de distintas estrategias y estilos de aprendizaje? 3) ¿Las variables académicas son capaces de introducir diferencias significativas en los estilos y estrategias de aprendizaje de los estudiantes universitarios? 4) ¿Los estilos y las estrategias de aprendizaje, influyen de modo significativo sobre el rendimiento académico de los estudiantes universitarios? Brindar respuesta a lo anterior resulta de interés en el diseño o modificación de los planes de estudio, y de los programas y metodologías de enseñanza específicos acordes con las características distintivas de los alumnos y de cada carrera o disciplina en particular. A la luz de lo anterior se plantean los siguientes objetivos: 1) Analizar diferencias estadísticamente significativas en las dimensiones de estilos y estrategias de aprendizaje según distintas variables sociodemográficas — sexo, edad, nivel educativo parental—. 2) Examinar la relación entre las diferentes dimensiones de estilos y estrategias de aprendizaje con el uso de recursos tecnológicos. 3) Estudiar diferencias estadísticamente significativas en las dimensiones de estilos y estrategias de aprendizaje según variables académicas — tipo de educación secundaria, carrera4) Analizar la influencia que los estilos y estrategias de aprendizaje tienen sobre el rendimiento académico de los estudiantes universitarios. 


\section{Método}

Se empleó un diseño transversal, correlacional, de diferencias entre grupos, y explicativo (Miles \& Banyard, 2007).

\section{Participantes}

Los datos se recogieron mediante un muestreo por conveniencia. Participaron 438 estudiantes universitarios de Buenos Aires $(59.8 \%$ varones; $40.2 \%$ mujeres) de diferentes carreras (17.1\% Psicología, 17.1\% Ingeniería Industrial, 16.4\% Física, 16\% Abogacía, 13.5\% Medicina, 11\% Computación, 8.9\% Ingeniería Electromecánica) de dos universidades públicas. Las edades variaban entre 19 y 36 años $(M=23.96 ; D E=3.30)$. Se optó por incluir en la muestra a aquellos estudiantes que al momento de recoger los datos se hallasen cursando por lo menos el segundo año de la carrera. Esta decisión obedece a la intención de trabajar con un grupo de alumnos con un grado mínimo de permanencia en la disciplina elegida por ellos inicialmente debido a que, según se conoce, el $58 \%$ del total de estudiantes que cursan el primer año abandona los estudios o cambia de carrera (Pintos, 2012). Se aplicó a todos los estudiantes un consentimiento informado previo a la administración de las pruebas.

\section{Medición}

La información sociodemográfica y académica se recogió a través de una encuesta que incluyó las variables sexo, edad, nivel educativo parental, cantidad de redes sociales que utiliza el estudiante frecuentemente, cantidad de dispositivos tecnológicos que emplea el educando cotidianamente, tipo de educación secundaria — bachiller o técnico-, carrera, antigüedad en años que lleva el estudiante en la carrera, número total de asignaturas aprobadas.

Los estilos de aprendizaje se evaluaron a través del Cuestionario Honey-Alonso de Estilos de Aprendizaje -CHAEA- (Alonso, Gallego \& Honey, 1994). Se aplicó la versión adaptada del instrumento para estudiantes universitarios de Buenos Aires (Freiberg-Hoffmann \& 
Fernández-Liporace, 2013). La herramienta, de breve administración, se compone de 28 ítems dicotómicos (afirmaciones) a los que los examinados deben responder según se hallen de acuerdo (+) o en desacuerdo (-). El instrumento permite evaluar cuatro estilos de aprendizaje, tres correspondientes al modelo de Kolb — Adaptador, Asimilador, Convergente - y uno al de Alonso et al. (1994) —Pragmático-, que describen diferentes preferencias de los sujetos para aprender. Esta versión del cuestionario, localmente adaptada, presenta evidencias de validez de contenido, facies y constructo —análisis de componentes principales y análisis factorial confirmatorio-, y apropiada consistencia interna para cada una de sus dimensiones teniendo en cuenta el número de ítems conservado por cada una de ellas (coeficientes KR20 entre .50 y .70).

Las estrategias de aprendizaje, por su parte, fueron evaluadas a través del Inventario de Estrategias de Aprendizaje y Estudio (LASSI) (Weinstein, Schulte \& Palmer, 1987). La versión del instrumento empleada corresponde a la validación efectuada con estudiantes universitarios de Buenos Aires (Freiberg-Hoffmann, Ledesma \& Fernández-Liporace, 2017). La misma cuenta con evidencias de validez de contenido, facies y de constructo —análisis de componentes principales y factorial confirmatorio- . Presenta además adecuada consistencia interna (coeficientes Alfa de Cronbach mayores a .75) y estabilidad temporal de las puntuaciones de cada dimensión. Se compone de 33 reactivos respondidos mediante una escala Likert de cinco opciones. Evalúa cinco tipos de estrategias siendo tales, Motivación —actitudes académicas y metas que guían al sujeto en el estudio-, Recursos para el Aprendizaje - técnicas empleadas por los alumnos para ayudarse a estudiar y facilitar el proceso de comprensión, adquisición y evocación de contenidos-, Habilidades para Jerarquizar la Información - pericia con la que cuenta el alumno para seleccionar la información importante que debe ser estudiada con mayor profundidad-, Aprendizaje Colaborativo — comunicación interpersonal orientada a mejorar el acceso a la información y contenidos de aprendizaje-, y Competencias para el Manejo de la Información 2.0 - estrategias vinculadas a la búsqueda, análisis, selección y comunicación de datos-. 


\section{Procedimiento}

La recolección de datos contó con los avales institucionales y de los profesores de cada unidad académica y se siguieron las consideraciones éticas señaladas por la APA para la investigación con seres humanos. La administración de los instrumentos se efectuó durante el horario habitual de clases, advirtiendo a los estudiantes acerca del carácter voluntario de su participación y ofreciéndoles la posibilidad de cesar de responder en cualquier instancia de la aplicación. Se administró además un consentimiento informado escrito y se garantizó la confidencialidad de los resultados y anonimato de los datos, anticipando asimismo que no habría una devolución individual. No se ofreció retribución económica ni de créditos académicos.

\section{Análisis de datos}

Los datos fueron analizados mediante el programa SPSS 21. Se aplicaron pruebas paramétricas en los casos en que los supuestos de normalidad y homocedasticidad se hubieran cumplido, empleando su variante robusta en aquellos en los que no. Se estimaron además los tamaños de los efectos considerando para su lectura los criterios de interpretación propuestos por Cohen (1977).

Con respecto a los análisis que incluyen las variables rendimiento académico y recursos tecnológicos resulta necesario mencionar que ambos conceptos carecen de una medida objetiva por lo que se torna necesario estimar índices representativos, aunque parciales de aquellos. El rendimiento académico se estimó adoptando la definición operacional propuesta por Tejedor-Tejedor (2003) de rendimiento inmediato en sentido amplio, que considera el rendimiento como éxito académico (finalizar una asignatura, curso, ciclo o titulación, en tiempo y forma). Para ello, se incluyeron en la encuesta académica dos ítems que posibilitan calcular el desempeño a partir del cociente entre el número de asignaturas aprobadas y la antigüedad en años que lleva cada estudiante cursando desde que ingresó a la carrera hasta la actualidad, obteniéndose como resultado un índice cuyo valor correlaciona positivamente con el 
rendimiento académico (De Miguel \& Arias, 1999). Esta modalidad de evaluación del desempeño académico es empleada en investigaciones recientes (e.g. Di Gresia, Porto, Ripani \& Sosa-Escudero, 2003; Ibarra \& Michalus, 2010; Porto \& Di Gresia, 2001).

Debido a los recursos tecnológicos y la amplitud que ese concepto entraña, es que se decidió en esta investigación circunscribirlo a la cantidad de redes sociales y dispositivos que los estudiantes emplean cotidianamente. Dicha selección se ha realizado en base a la relevancia que han demostrado poseer ambas características dentro del ámbito universitario (Cantilo, Roura \& Sánchez, 2012; Meso, Pérez \& Mendiguren, 2011). Esto permitió calcular una medida relativa y parcialmente representativa de lo que se conoce habitualmente como recursos tecnológicos que, por su puesto, abarca una gran cantidad de aspectos tales como el empleo de plataformas virtuales, sistema de almacenamiento compartido - la nube-, sistemas de gestión de conocimientos —wikis, blogs-, y software educativo, entre otros (Colorado-Aguilar \& Edel-Navarro, 2012). Se sumó entonces, tanto el número de redes sociales como la cantidad de dispositivos que habitualmente emplean los estudiantes, obteniendo una medida cuantitativa de los recursos tecnológicos.

\section{Resultados}

\section{Estilos y estrategias de aprendizaje según variables sociodemográficas}

Se comenzó por analizar diferencias estadísticamente significativas en las dimensiones de estilos y estrategias de aprendizaje según sexo. Se aplicó la prueba $t$ para muestras independientes observándose diferencias en los estilos Adaptador $\left(M_{\text {varones }}=4.06 ; D E=1.09 ; M_{\text {mujeres }}=3.79\right.$; $D E=1.25 ; p=.02 ; d=.23)$ y Pragmático $\left(M_{\text {varones }}=1.96 ; D E=1.22\right.$; $\left.M_{\text {mujeres }}=1.56 ; D E=1.10 ; p=.001 ; d=.34\right)$ ambas a favor de los varones. Asimismo se registraron diferencias significativas en las estrategias de Motivación $\left(M_{\text {varones }}=24.50 ; D E=3.59 ; M_{\text {mujeres }}=26.71\right.$; 
$D E=3.26 ; p<.001 ; d=.64)$ y Habilidades para Jerarquizar la Información $\left(M_{\text {varones }}=17.25 ; D E=2.87 ; M_{\text {mujeres }}=17.82 ; D E=2.64 ; p=.04\right.$; $d=.20$ ), favorables a las mujeres.

Seguidamente se procedió a estudiar diferencias significativas en las dimensiones de estilos y estrategias de aprendizaje según edad. Para ello se recategorizó la variable edad en estudiantes jóvenes (19 a 23 años) y mayores (24 a 36 años) tomándose como punto de corte el percentil 50. Luego se aplicó una prueba $t$ para muestras independientes verificándose diferencias significativas para el estilo Asimilador $\left(M_{\text {jovenes }}=8.18\right.$; $\left.D E=1.55 ; \mathrm{M}_{\text {mayores }}=7.85 ; D E=1.74 ; p=.04 ; d=.20\right)$ y la estrategia de Motivación $\left(M_{\text {jovenes }}=25.89 ; D E=3.46 ; M_{\text {mayores }}=24.78 ; D E=3.71\right.$; $p=.001 ; d=.30)$ ambas favorables a los estudiantes jóvenes.

Posteriormente se examinaron las dimensiones de estilos y estrategias según el nivel educativo parental, comparándose estudiantes según sus padres, por un lado, y madres por el otro, hubieran obtenido o no un título de educación superior. Comenzando por el análisis según nivel educativo del padre se registró una diferencia en el estilo Convergente a favor de los estudiantes con padres con título superior $\left(M_{\text {ctitulo }}=5.31\right.$; $D E=1.78 ; M_{\text {stitulo }}=4.73 ; D E=1.84 ; p=.001 ; d=.32$ ). Respecto del examen concerniente al nivel educativo de la madre se registraron diferencias significativas a favor de aquellos estudiantes cuyas madres poseyeran titulación superior en el estilo Asimilador $\left(M_{c \text { titulo }}=8.18\right.$; $\left.D E=1.56 ; M_{\text {stitulo }}=7.86 ; D E=1.73 ; p=.05 ; d=.19\right)$ y en las estrategias de Motivación $\left(M_{\text {cltitulo }}=25.97 ; D E=3.52 ; M_{\text {stitulo }}=24.74 ; D E=3.63\right.$; $p=.00 ; d=.34)$ y Recursos para el Aprendizaje $\left(M_{c \text { titulo }}=14.06\right.$; $\left.D E=2.94 ; M_{\text {stitulo }}=13.42 ; D E=2.95 ; p=.02 ; d=.21\right)$.

\section{Relación entre estilos y estrategias de aprendizaje con el uso de recursos tecnológicos}

Se correlacionó la medida de los recursos tecnológicos con las dimensiones de estilos y estrategias verificándose varias correlaciones estadísticamente significativas y positivas aunque débiles, con los estilos Convergente $(r=.11 ; p=.03)$ y Adaptador $(r=.10 ; p=.03)$ por un 
lado, y las estrategias de Aprendizaje Colaborativo $(r=.12 ; p=.01)$, y Recursos para el Aprendizaje $(r=.14 ; p=.005)$, por otro.

\section{Estilos y estrategias de aprendizaje según variables académicas}

Se procedió a analizar diferencias estadísticamente significativas según el tipo de educación secundaria que hubieran cursado los estudiantes universitarios. Para ello se tomó la clasificación efectuada por el Ministerio de Educación de la Nación (2016) que agrupa las titulaciones en dos categorías mayores: bachiller y técnico. Se observaron diferencias estadísticamente significativas en las estrategias de Motivación y Recursos para el Aprendizaje, la primera a favor de los estudiantes con título de bachiller $\left(M_{\text {bachiller }}=25.60 ; \mathrm{DE}=3.65 ; M_{\text {técnico }}=24.44\right.$; $D E=3.33 ; p=.01 ; d=.33)$ y la segunda, favorable a los egresados de la orientación técnica $\left(M_{\text {bachiller }}=13.59 ; D E=2.98 ; M_{\text {técnico }}=14.42\right.$; $D E=2.78 ; p=.02 ; d=.28)$.

Luego se examinaron diferencias en las dimensiones de estilos y estrategias según carrera. Para ello se aplicó una prueba ANOVA de una vía con contraste post hoc Tukey. Se observaron múltiples diferencias estadísticamente significativas en todas las dimensiones a excepción del estilo Asimilador (Tabla 1).

\section{Influencia de los estilos y estrategias de aprendizaje sobre el rendimiento académico}

Para analizar la influencia de los estilos y estrategias de aprendizaje sobre el rendimiento académico se ejecutó un análisis multinivel, que parte de la presunción de que los sujetos de un mismo grupo (estudiantes universitarios) no son independientes entre sí, sino que comparten ciertas características aun cuando puedan clasificarse en subgrupos (carreras). Este análisis posibilita la detección de efectos comunes que las variables independientes tienen sobre la dependiente en los universitarios, como también los efectos aleatorios atribuibles a la especificidad de cada carrera (Pardo, Ruiz \& San Martín, 2007; Sora, Caballer \& Peiró, 2011). 


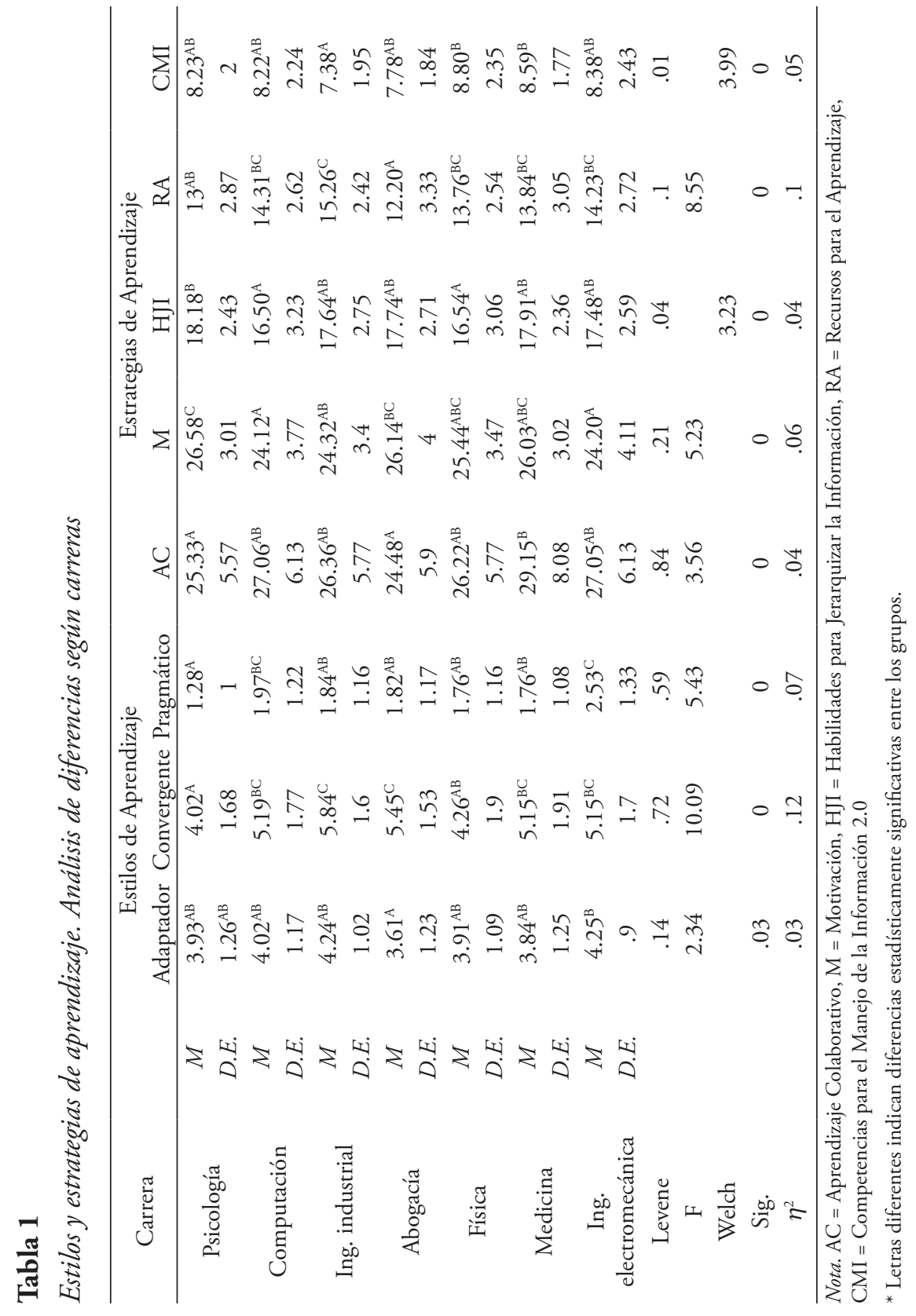


Estilos y estrategias de aprendizaje en estudiantes universitarios de Buenos Aires / Freiberg et al.

Para comenzar, se estimó un modelo nulo con el propósito de comparar más adelante el efecto atribuible a las diferencias entre carreras. Para ello se empleó como variable de agrupación a las carreras y al rendimiento académico como variable dependiente. Posteriormente, se estimó un modelo de un factor de efectos aleatorios (EA) con el fin de indagar la influencia de los estilos y estrategias de aprendizaje sobre el rendimiento académico, y si esos efectos dependen en gran medida de la especificidad de cada carrera o pueden interpretarse del mismo modo para la totalidad de los estudiantes universitarios. Para ello se seleccionaron los cuatro estilos - Asimilador, Adaptador, Convergente, Pragmático- y las cinco estrategias —Aprendizaje Colaborativo, Motivación, Habilidades para Jerarquizar la Información, Recursos para el Aprendizaje, Competencias para el Manejo de la Información 2.0 - como covariables. Se adoptó también la clasificación de los estudiantes por carrera como variable de agrupación. Con todas estas variables se planteó un modelo de 2 niveles que fue estimado mediante el método de máxima verosimilitud restringida (MVR) dada la robustez del mismo frente al bajo tamaño muestral que representaba a cada carrera en la muestra total (Heck, Thomas \& Tabata, 2011; Martínez-Garrido \& Murillo, 2014).

Los resultados presentados en la tabla 2 permiten comparar el modelo nulo con el de efectos aleatorios. Comenzando por el nivel que integra a la totalidad de los estudiantes se observó, mediante el análisis de los residuos tanto para el modelo nulo como para el EA, un valor del estadístico Wald significativo, indicando ello que el rendimiento académico varía entre todos los estudiantes, incluso entre los educandos de las mismas carreras. Al comparar las estimaciones de ambos modelos se registró una leve disminución en la varianza del modelo EA (2.27) en comparación con el nulo (2.59). Tal diferencia implica que las dimensiones de estilos y estrategias incluidas en el modelo EA ayudarían a disminuir en proporción relativa la variabilidad del rendimiento académico en el conjunto de los estudiantes.

Pasando ahora al nivel que agrupa a los estudiantes en las distintitas carreras se observó, en primer lugar, la ausencia de variabilidad entre 
carreras en relación al rendimiento académico ya que el estadístico Wald no resultó significativo. La falta de significación de este estadístico parecería indicar que no existirían tales diferencias, llevando a pensar que las dimensiones de estilos y estrategias serían las características principales que posibilitarían explicar para todas las especialidades académicas por igual el desempeńo estudiantil. Puesto que este resultado no parece coherente con la teoría ni con el resultado obtenido en el análisis del nivel anterior, y teniendo en cuenta que el estadístico Wald es considerado como muy conservador cuando se trabaja con muestras pequeñas, es que se decidió estimar según la sugerencia de otros investigadores el estadístico de razón de verosimilitud, robusto frente a dicha situación (Pardo, Ruiz \& San Martín, 2007; Stroup, 2013). Para ello se empezó por comparar los índices -2 log. de verosimilitud restringida (-2LL), de ambos modelos (nulo y EA) (Heck, Thomas \& Tabata, 2011). Puesto que valores bajos son indicadores de una mayor variabilidad entre carreras, es que puede afirmarse que los rendimientos de los educandos de las distintas especialidades varían aun cuando sean controladas las dimensiones de los estilos y las estrategias de aprendizaje. Para conocer si tal variación es estadísticamente significativa se procedió a calcular el valor de chi cuadrado restando al estadístico -2LL del modelo nulo (2199.27) el valor del estadístico -2LL del modelo AE (1597.68), lo que da un valor de 601.42. Luego se fijó el número de grados de libertad en 10 en base a la diferencia en la cantidad de parámetros que integraba cada modelo (Pardo, Ruiz \& San Martín, 2007). Estos datos permitieron estimar el p-valor que alcanzó un grado óptimo $(p<.01)$ indicando ello que la variabilidad entre carreras es estadísticamente significativa. Ulteriormente se intentó averiguar el porcentaje de varianza total del rendimiento académico que se debe a las diferencias entre las carreras luego de controlar las dimensiones de estrategias y estilos de aprendizaje. Para ello se calculó el coeficiente de correlación intraclase (CCI) que arrojó un valor .19, lo que se traduce en una explicación del 19\% de la varianza total del rendimiento académico atribuible a las diferencias entre carreras. Por otra parte, se estimó otro CCI para conocer el porcentaje de varianza del rendimiento académico que las dimensiones de estilos y estrategias consiguen explicar. Se obtuvo 
Estilos y estrategias de aprendizaje en estudiantes universitarios de Buenos Aires / Freiberg et al.

así un valor de .47 , que implica que el $47 \%$ de la varianza total del desempeño estudiantil se explica por las dimensiones de estilos y estrategias.

\section{Tabla 2}

Estimación de los parámetros de covarianza

\begin{tabular}{|c|c|c|c|c|c|c|c|c|}
\hline & Parámetro & & $\begin{array}{c}\text { Estima- } \\
\text { ción }\end{array}$ & $E T$. & Wald Z & $p$ & $\begin{array}{c}\mathrm{IC}^{\mathrm{a}} \\
{[95 \%]}\end{array}$ & $-2 L^{b}$ \\
\hline \multirow{2}{*}{$\mathrm{MN}^{\mathrm{c}}$} & Residuos & & 2.59 & .18 & 14.44 & .00 & {$[2.26 ; 2.96]$} & \multirow{2}{*}{2199.27} \\
\hline & Carrera & Var. & .50 & .32 & 1.59 & .11 & {$[.15 ; 1.72]$} & \\
\hline \multirow[b]{2}{*}{$\mathrm{MEA}^{\mathrm{d}}$} & Residuos & & 2.27 & .16 & 14.25 & .00 & {$[1.98 ; 2.60]$} & \multirow[b]{2}{*}{1597.68} \\
\hline & $\begin{array}{l}\text { Intersección } \\
{[\text { sujeto = carrera }]}\end{array}$ & Var. & .56 & .35 & 1.61 & .10 & {$[.17 ; 1.90]$} & \\
\hline
\end{tabular}

Nota. a Intervalo de Confianza, b -2 log. de la verosimilitud restringida, c Modelo Nulo, d Modelo de Efectos Aleatorios

Pasando ahora al análisis de los parámetros estimados de efectos fijos del modelo, los mismos se resumen en la tabla 3. Como puede verse, el valor de la intersección fue positivo y significativo lo que estaría indicando que el rendimiento académico medio entre carreras no es el mismo o bien es distinto de cero. Por otro lado, las estimaciones correspondientes a las covariables mostraron que de las nueve variables incluidas en el modelo solo cinco lograron explicar de modo significativo el rendimiento académico, ellas fueron el estilo Pragmático, y las estrategias de Aprendizaje Colaborativo, Motivación, Habilidades para Jerarquizar la Información y Competencias para el Manejo de la Información 2.0. Como puede verse algunas estimaciones fueron positivas y otras negativas, pudiéndose interpretar que el rendimiento académico se incrementa en .13, .03, .14, .07 y .09 cuando las variables de estilo Pragmático, y las estrategias de Aprendizaje Colaborativo y Motivación se incrementan en un punto y las de Habilidades para Jerarquizar la Información y Competencias para el Manejo de la Información 2.0 decrecen en un punto respectivamente. 


\section{Tabla 3}

Estimación de los parámetros de efectos fijos del modelo de efectos aleatorios

\begin{tabular}{lcccccc}
\hline Parámetro & Estimación & ET & Gl & $t$ & $p$ & $\begin{array}{c}\text { IC } \\
{[95 \%]}\end{array}$ \\
\hline Intersección & 2.51 & .85 & 229.60 & 2.96 & .00 & {$[.84 ; 4.18]$} \\
Asimilador & -.02 & .05 & 407.96 & -.39 & .69 & {$[-.12 ; .08]$} \\
Convergente & -.06 & .04 & 410.28 & -1.48 & .13 & {$[-.15 ; .02]$} \\
Adaptador & .03 & .06 & 408.07 & .55 & .58 & {$[-.09 ; .17]$} \\
Pragmático & .13 & .06 & 409.05 & 1.98 & .04 & {$[.00 ; .27]$} \\
AC & .03 & .01 & 407.91 & 3.04 & .00 & {$[.01 ; .06]$} \\
M & .14 & .02 & 410.34 & 6.19 & .00 & {$[.09 ; .18]$} \\
HJI & -.07 & .02 & 408.73 & -2.58 & .01 & {$[-.12 ;-.01]$} \\
RA & -.03 & .02 & 411.24 & -1.23 & .21 & {$[-.09 ; .02]$} \\
CMI & -.09 & .03 & 408.20 & -2.48 & .01 & {$[-.17 ;-.02]$} \\
\hline
\end{tabular}

\section{Discusión}

Este estudio se propuso conocer el modo en que los estilos y las estrategias de aprendizaje se presentan en los estudiantes universitarios, para así planificar acciones en diferentes planos educativos -institucional, docencia, estudiantil, tutorial, entre otros- tendientes a mejorar la calidad de los aprendizajes.

Comenzando por el análisis de los estilos y las estrategias de aprendizaje según variables sociodemográficas, se observó primeramente que los varones presentan una mayor preferencia por los estilos Adaptador y Pragmático, comparados con las mujeres. Ambos estilos se relacionan con modalidades de aprendizaje caracterizadas por la acción y no tanto por la reflexión. Así, los adaptadores se describen como sujetos que prefieren resolver situaciones novedosas haciendo uso de sus intuiciones 
y sentimientos antes que del análisis lógico. Por su parte, los pragmáticos se destacan por su interés para poner en práctica toda idea que les resulte atractiva, resolviendo de modo inmediato y directo cualquier situación problemática. Los estudios previos realizados al respecto han informado que en las mujeres prima la reflexión y teorización por sobre la inmediata puesta a prueba de las ideas, mientras que sus pares masculinos exhiben la tendencia opuesta (Aguilar, 2010; López-Aguado, 2011). De este modo, los hallazgos en cuanto a estas dimensiones resultan coherentes con los antecedentes mencionados.

Por otro lado, las mujeres utilizan con mayor frecuencia que los varones las estrategias de Motivación y Habilidades para Jerarquizar la Información. Esto implicaría que ellas se destacan por sus actitudes académicas y metas que guían su estudio, a la vez que parecen contar con una mayor pericia para seleccionar la información importante que debe ser aprendida en profundidad. Existen estudios que verifican la diferencia favorable para las mujeres, tanto respecto del uso más frecuente de la estrategia de Motivación (Griffin, MacKewn, Mosare \& VanVuren, 2012; Ku \& Chang, 2011; Marland, Dearlove \& Carpenter, 2015) como de la de Habilidades para Jerarquizar la Información (Aguilar, 2010; Jouhari, Haghani \& Changiz, 2016). Con respecto a la Motivación suele argumentarse que las mujeres, a diferencia de los varones, tienen una mayor predisposición a la lectura y a completar las actividades académicas en tiempo y forma, además de tomar muy serio los estudios y de preocuparse por su exitosa finalización (Ross \& Powell, 1999). Por otro lado, la mayor frecuencia de uso en las mujeres respecto de los varones en cuanto a la estrategia Habilidades para Jerarquizar la Información podría explicarse por el empleo que hacen de las estrategias de Motivación, ya que suele afirmarse que la capacidad para seleccionar ideas principales se asocia positivamente con la actitud y el compromiso académico adoptado por el estudiante (Loomis, 2000). De este modo, sería interesante en futuras investigaciones analizar la relación entre ambas estrategias a fin de poner a prueba esta hipótesis.

Con relación al análisis según grupo etario, se registraron diferencias en el estilo Asimilador y la estrategia de Motivación, ambas 
favorables a los estudiantes jóvenes, resultado que abona el obtenido en otras investigaciones (Aguilar, 2010; Gargallo, Almerich, SuárezRodríguez \& García-Félix, 2012). De acuerdo con esto los educandos de menor edad tienen la cualidad de manejar y comprender una amplia variedad de información, ya que disfrutan del análisis de conceptos abstractos buscando la coherencia y validez lógica de las ideas —estilo Asimilador- Asimismo, presentan diversas estrategias motivacionales que se reflejan en actitudes académicas y metas que los guían en el estudio, asumiendo la responsabilidad de resolver las tareas necesarias para acceder al éxito académico. Debe aclararse que esta fuerte diferenciación que se da entre estudiantes jóvenes y mayores resulta lógica si se considera que, en general, los educandos de menor edad se concentran en instancias iniciales de las carreras, mientras que los mayores lo hacen en momentos intermedios o finales. En este sentido, es posible hipotetizar que ambas cualidades - estilo Asimilador y estrategia de Motivación - son imprescindibles cuando el estudiante ingresa al sistema universitario, ya que debe contar con aquellos recursos que le permitan percibir e incorporar toda clase de información, de modo tal de lograr una correcta adaptación al sistema, persistiendo en el mismo. No obstante, tales características deberían ir modificándose a lo largo del ciclo académico para adecuarse a las particularidades de cada carrera. Es posible que, avanzando en el trayecto académico, el alumno ya no necesite de tales estrategias motivacionales empleando otros recursos cognitivos y metacognitivos que comienzan a ser demandados por instancias ulteriores de cada carrera. Alternativamente, también podría pensarse que el gradual desuso de estrategias motivacionales pueda explicarse por una progresiva desmotivación a medida que se progresa en el plan de estudios, producto de las vicisitudes planteadas por el sistema. Sería interesante, en futuros trabajos, efectuar un estudio longitudinal que ponga a prueba estas hipótesis.

Pasando ahora al análisis según nivel educativo parental, varias investigaciones dan cuenta de la mayor posesión de recursos para el aprendizaje — cognitivos, metacognitivos, personológicos- de aquellos estudiantes cuyos padres poseen títulos académicos superiores. 
Esto se explica comúnmente aludiendo a que tales padres suelen inculcar en sus hijos hábitos de estudio, el placer por las tareas académicas, la curiosidad, la perseverancia, y la tolerancia a la frustración, entre otros aspectos útiles en la vida universitaria (Garbanzo-Vargas, 2007; Lee, Sax, Kim \& Hagedom, 2004; Porcel, Dapozo \& López, 2010). Según los resultados de la presente investigación, los estilos y las estrategias de aprendizaje también se verían afectados por el tipo de educación parental. Así podría hipotetizarse que los padres con título superior tenderían a estimular el estilo Convergente de aprendizaje, mientras que las madres con el mismo grado académico fomentarían en sus hijos tanto el estilo Asimilador como las estrategias de Motivación y Recursos para el Aprendizaje. Como puede apreciarse el nivel educativo de la madre parecería ser más relevante a la hora de estimular en los hijos el desarrollo de habilidades cognitivas y metacognitivas. En este sentido, es reconocida la mayor importancia del nivel educativo de la madre por sobre el del padre en cuestiones relativas al aprendizaje. Según se argumenta, a diferencia de los padres, las madres con mayor nivel educativo se interesan más por el devenir académico de los hijos, fomentan una actitud positiva hacia el estudio, se preocupan por su desempeño y su continuidad en el ámbito escolástico (Castejón \& Pérez, 1998; Marchesi, 2000; Morales, 1999).

Hasta acá se han examinado algunas variables sociodemográficas intentando detectar las preferencias para aprender de los estudiantes según esas características. Estas diferencias orientan sobre el perfil de aprendizaje de los educandos, permitiendo la planificación de diferentes prácticas que beneficien la asimilación de conocimientos y, por ende, mejores aprendizajes.

Pasando ahora al empleo que los estudiantes hacen de los recursos tecnológicos, se halló que su uso se relaciona de manera significativa y positiva con los estilos Convergente y Adaptador por un lado, y con las estrategias de Aprendizaje Colaborativo y Recursos para el Aprendizaje, por otro. Con respecto a los estilos - Convergente y Adaptador-, ambos presentan habilidades orientadas a la resolución de problemas, tarea que requiere de la utilización de diversos recursos 
tanto tecnológicos, como no tecnológicos. El Convergente comprende destrezas relacionadas con la toma de decisiones como por ejemplo, el análisis cuantitativo, la fijación de objetivos y el empleo de tecnología. El Adaptador, por su parte, incluye habilidades para la acción, tales como liderazgo e iniciativa (Kolb \& Kolb, 2005). Respecto de las estrategias, resulta lógica la relación con el Aprendizaje Colaborativo, ya que esta dimensión se vincula con el empleo de redes sociales y la comunicación entre alumnos durante el proceso de aprendizaje, siendo ello requerido principalmente en modalidades de enseńanza e-learning y b-learning (Morán, 2012). En cuanto a las estrategias de Recursos para el Aprendizaje, ellas incluyen técnicas empleadas por los educandos para ayudarse a estudiar y facilitar el proceso de aprendizaje. En este sentido, el empleo de los recursos tecnológicos parece ser un factor que participa en la comprensión, adquisición y evocación de contenidos (Stover, Uriel \& Fernández-Liporace, 2012). Debe destacarse, por otro lado, la falta de relación significativa entre las estrategias de Competencias para el Manejo de la Información 2.0 y los recursos tecnológicos, aspecto que resulta llamativo. Según esto, las técnicas vinculadas a la búsqueda, análisis, selección y comunicación de datos en medios tecnológicos no se encontrarían asociadas a la cantidad de herramientas que el estudiante emplea. En otras palabras, la cantidad de recursos no se condice necesariamente con la calidad en el uso de los mismos. Este aspecto resulta interesante, sobre todo a la hora de implementar políticas educativas orientadas a facilitar el acceso a las nuevas tecnologías. Tal decisión debería acompañarse de propuestas pedagógicas que instruyan a docentes y educandos en el empleo adecuado de tales herramientas, uso que redunde luego en un óptimo aprovechamiento de las mismas. Si bien los estudiantes utilizan recursos tecnológicos en su vida diaria para comunicarse, o bien para buscar información cotidiana —una dirección, una institución, o el significado de una palabra, entre otros- parece evidente que tales habilidades no se ajustan apropiadamente a las requeridas por el ámbito académico. En esta dirección, la repentina inclusión de medios digitales en la universidad torna necesario que los diferentes actores del sistema —instituciones, profesores, 
psicólogos educativos, entre otros - planifiquen mancomunadamente acciones tendientes a formar competencias para el adecuado manejo académico de las nuevas tecnologías, a fin de fomentar la apropiada discriminación de información relevante y válida en contextos escolásticos.

En relación con el análisis de las variables académicas, se advierte, respecto de la titulación de nivel medio que los estudiantes que cursaron bachilleratos, presentan un mayor número de estrategias de Motivación, mientras que aquellos con título técnico se destacan en el uso de Recursos para el Aprendizaje. Esto quiere decir que los bachilleres se caracterizan por establecer metas de estudio, leer textos, preparar clases y finalizar las tareas a tiempo. En cambio, los egresados de la educación técnica se distinguen por emplear recursos gráficos tales como cuadros, mapas conceptuales, resaltados, entre otros. Este resultado parecería indicar el tipo de estrategia que ha sido reforzada en los estudiantes en el nivel educativo medio según la especialidad. Esta información cobra relevancia a la hora de saber si un alumno contará con las estrategias necesarias para aprehender la información de una carrera en particular. Ello se complementa con los resultados derivados del análisis de diferencias según carrera. De acuerdo con ellos, los estudiantes de Psicología emplean frecuentemente estrategias de Motivación, mientras que los de Ingeniería Industrial usan más Recursos para el Aprendizaje. Esto permite hipotetizar que los bachilleres se gradúan del nivel medio con estrategias de aprendizaje entrenadas que son compatibles con la carrera de Psicología, ocurriendo lo mismo entre los egresados de la educación técnica respecto de la carrera Ingeniería Industrial. Resta analizar en futuras investigaciones, al interior de cada disciplina universitaria, la medida en que dicha coincidencia beneficiaría el rendimiento académico de los estudiantes, así como ampliar las muestras analizadas a mayor cantidad de carreras.

Continuando con el análisis de diferencias entre carreras, puede intentarse describir brevemente los perfiles estudiantiles de estilos y estrategias de aprendizaje, según aquellas dimensiones que distinguen a los alumnos de una disciplina en particular respecto del resto. Se destaca, así, 
para estudiantes de Ingeniería Electromecánica la presencia acentuada de los estilos Adaptador y Pragmático. Para los de Abogacía se registra una baja preferencia por el estilo Adaptador y de las estrategias de Recursos para el Aprendizaje, junto con una presencia marcada del estilo Convergente. Los estudiantes de Psicología por su parte, manifiestan emplear una mayor variedad de estrategias de Motivación, junto con una baja preferencia por los estilos Convergente, Pragmático y las estrategias de Aprendizaje Colaborativo y Habilidades para Jerarquizar la Información. Los estudiantes de Computación se destacan por utilizar tanto pocas estrategias de Motivación como de Habilidades para Jerarquizar la Información. En cambio los de Ingeniería Industrial se caracterizan por la preferencia por el estilo Convergente y el empleo de estrategias de Recursos para el Aprendizaje, y un escaso uso de estrategias vinculadas con las Competencias para el Manejo de la Información 2.0. Los de Física se distinguen por su alta preferencia en cuanto estrategias de Competencias para el Manejo de la Información 2.0 y por el esporádico uso de Habilidades para Jerarquizar la Información. Por último, los estudiantes de Medicina se caracterizan por un frecuente empleo de estrategias de Aprendizaje Colaborativo y Competencias para el Manejo de la Información.

Los perfiles mencionados orientan acerca de las preferencias específicas del estudiantado de cada carrera analizada para percibir y procesar la información, como también sobre las acciones concretas que se emprenden para resolver las tareas académicas (Kolb \& Kolb, 2005). Así, es posible conocer aquellas cualidades cognitivas y metacognitivas que facilitarían el proceso de aprendizaje. La utilidad de esta descripción de perfiles radica en la posibilidad docente de adecuar sus prácticas pedagógicas a las necesidades del alumnado, mejorando así la incorporación de información novedosa y, consecuentemente, la calidad educativa.

Pasando ahora al estudio multinivel del rendimiento académico, los resultados obtenidos muestran que, si bien todos los estudiantes de todas las carreras difieren en cuanto a su rendimiento académico, estas diferencias se acortan al introducir las dimensiones de estilos y estrategias de aprendizaje. Si bien no es posible conocer la importancia 
relativa de cada dimensión en cada carrera, sí se observa que el modelo de estilos y estrategias planteado para todas las disciplinas académicas consigue explicar el $47 \%$ de la variabilidad del rendimiento de los estudiantes, quedando un $19 \%$ de la varianza total explicada por las diferencias entre carreras. Cabe resaltar que, del $47 \%$ de la varianza explicada por el modelo, la variable que realiza el mayor aporte es la estrategia de Motivación, seguida por el estilo Pragmático y las estrategias de Habilidades para Jerarquizar la Información, Competencias para el Manejo de la Información 2.0, y Aprendizaje Colaborativo. Asimismo resulta conveniente destacar que estos aportes son algunos positivos y otros negativos, lo que significa que el rendimiento estudiantil se incrementa cuando las variables de estilo Pragmático y las estrategias de Aprendizaje Colaborativo y Motivación se utilizan con mayor frecuencia, y las estrategias de Habilidades para Jerarquizar la Información y Competencias para el Manejo de la Información 2.0 se emplean en baja medida. Con respecto a las asociaciones negativas de las dos últimas estrategias cabe recordar que como se mencionó en el apartado teórico una de las posibles formas de mejorar los aprendizajes consiste en detectar las estrategias responsables del bajo rendimiento e intentar mejorarlas (Beltrán, 2003). En este sentido, se torna cada vez más necesario entrenar a los alumnos en las Competencias para el Manejo de la Información 2.0 de modo tal que sean capaces de buscar, analizar, seleccionar y comunicar la información proveniente de los distintos dispositivos tecnológicos. La accesibilidad a la información con la que contamos actualmente requiere de parte de los estudiantes del desarrollo de esta clase de estrategias que les permitan distinguir la información confiable de la que no lo es o, en otras palabras, adquirir las competencias necesarias para filtrar correctamente la información (Eco, 2010). Multiplicidad de recursos e inundación de información no necesariamente implican un adecuado manejo de ambos aspectos. En esta dirección, también se requiere mejorar las estrategias vinculadas con las Habilidades para Jerarquizar la Información a modo tal de garantizar un exitoso proceso de selección, ya sea con respecto a relevancia, a su calidad y confiabilidad. 
Con respecto a las limitaciones del estudio, resulta interesante destacar que de acuerdo con lo argumentado en el apartado metodológico, se ha decidido no incluir en la muestra a aquellos alumnos que se encontraban cursando el primer ańo del ciclo universitario, a fin de trabajar con estudiantes con un mínimo grado de permanencia en la carrera por ellos elegida. Esta selección de la muestra imposibilitó efectuar comparaciones entre estudiantes que recién inician sus estudios versus aquellos que se encuentran en instancias avanzadas, lo que hubiera permitido observar diferencias entre ambos grupos de educandos. Se espera así, en futuras investigaciones, recoger información de ingresantes a fin de realizar el contraste entre ambos grupos. Además sería interesante complementar esta información mediante estudios longitudinales que vuelvan factible observar la evolución de los estilos y las estrategias de aprendizaje a lo largo del ciclo universitario.

Por otra parte, debe mencionarse entre las limitaciones que los estudiantes que formaron parte de la muestra pertenecían todos ellos a instituciones universitarias públicas. Si bien, de acuerdo con datos oficiales (Ministerio de Hacienda, Buenos Aires Ciudad, 2016) el 70\% de los alumnos de Buenos Aires cursan sus estudios en universidades públicas, correspondiendo el $30 \%$ restante al ámbito privado, sería importante incluir en el futuro educandos del ámbito privado en lo posible mediante un muestreo por cuotas que respete esta proporción con el fin de trabajar con una muestra más representativa de la población estudiantil local.

El presente trabajo se ha propuesto identificar estilos y estrategias de aprendizaje presentes en los estudiantes universitarios con distintas características sociodemográficas y académicas y, al mismo tiempo, conocer cuáles de tales estilos y estrategias efectúan un aporte significativo a la explicación del rendimiento académico. Los resultados obtenidos describen sus preferencias para aprender y los estilos y estrategias vinculadas con el éxito académico. Esto posibilitará a los profesionales psicólogos del ámbito educativo trabajar con los docentes en la planificación de actividades pedagógicas que, 1) fomenten el desarrollo, o mejoren las estrategias que son relevantes para el devenir 
académico estudiantil y 2) adapten sus estrategias didácticas y programas a los estilos de aprendizaje de los alumnos para que estos logren procesar la información con mayor facilidad y eficacia. Ambos objetivos requieren del diseño de materiales novedosos tanto en lo que refiere a la presentación y organización de las clases, como también respecto de las tareas planteadas, previo entrenamiento de quienes se hallan a cargo de estas actividades docentes.

El diseño y puesta en marcha de nuevas prácticas pedagógicas es un desafío que debe llevarse a cabo para mejorar los aprendizajes. Si bien esta cuestión excede el propósito de la presente investigación, la intención última reside en presentar algunas características cognitivas y metacognitivas que deben considerarse de cara a la reestructuración de las actuales metodologías educativas. Se espera, así, que futuras investigaciones planifiquen y pongan a prueba nuevas actividades tomando como base los resultados aquí presentados.

\section{Referencias}

Abidin, M. J., Rezaee, A. A., Abdullah, H. N. \& Singh, K. K. (2011). Learning styles and overall academic achievement in a specific educational system. International Journal of Humanities and Social Sciences, 1(10), 143-152.

Aguilar, M. C. (2010). Estilos y estrategias de aprendizaje en jóvenes ingresantes a la universidad. Revista de Psicología, 28(2), 207-226. AlKhasawneh, E. (2013). Using VARK to assess changes in learning preferences of nursing students at a public university in Jordan: Implications for teaching. Nurse Education Today, 33(12), 15461549. https://doi.org/10.1016/j.nedt.2012.12.017

Allueva-Torres, P. \& Bueno-García, C. (2011). Estilos de aprendizaje y estilos de pensamiento en estudiantes universitarios. Aprender a aprender y aprender a pensar. ARBOR Ciencia, Pensamiento y Cultura, 187(3), 261-266. https://doi.org/10.3989/arbor.2011. Extra-3n3155 
Almigbal, T. H. (2015). Relationship between the learning style preferences of medical students and academic achievement. Saudi Medical Journal, 36(3), 349-355.https://doi.org/10.15537/ smj.2015.3.10320

Alonso, C. M., Gallego, D. J. \& Honey, P. (1994). Los estilos de aprendizaje. Procedimientos de diagnóstico y mejora. Bilbao: Mensajero. Aragón-García, M. \& Jiménez-Galán, Y. (2009). Diagnóstico de los estilos de aprendizaje en los estudiantes: Estrategia docente para elevar la calidad educativa. Revista de Investigación Educativa, 9, 1-21.

Bahamón, M., Vianchá, M., Alarcón, L. \& Bohórquez, C. (2012). Estilos y estrategias de aprendizaje: una revisión empírica y conceptual de los últimos diez años. Pensamiento Psicológico, 10(1), 129-144.

Bahamón, M., Vianchá, M., Alarcón, L. \& Bohórquez, C. (2013). Estilos y estrategias de aprendizaje relacionados con el logro académico en estudiantes universitarios. Pensamiento Psicológico, 11(1), 115-129.

Beltrán, J. A. (2003). Estrategias de aprendizaje. Revista de Educación, 332, 55-73.

Biggio, M., Vázquez, S. \& García, S. (2015). Deserción en estudiantes de nuevo ingreso a carreras de Diseño. El caso de la Universidad de Buenos Aires, Argentina. Actualidades Investigativas en Educación, 15(1), 1-23.

Biggs, J. B. (1988). The role of metacognition in enhancing learning. Australian Journal of Education, 32,127-138. https://doi. org/10.1177/000494418803200201

Blumen, S., Rivero, C. \& Guerrero, D. (2011). Universitarios en educación a distancia: estilos de aprendizaje y rendimiento académico. Revista de Psicología, 29(2), 225-243.

Borracci, R. A. \& Arribalzaga, E. B. (2015). Estilos de aprendizaje de Kolb en estudiantes de medicina. Medicina, 75, 73-80.

Broadbent, J. \& Poon, W. L. (2015). Self-regulated learning strategies $\&$ academic achievement in online higher education learning environments: A systematic review. The Internet 
Estilos y estrategias de aprendizaje en estudiantes universitarios de Buenos Aires / Freiberg et al.

and Higher Education, 27, 1-13. https://doi.org/10.1016/j. iheduc.2015.04.007

Caballero-Vargas, M. C. (2015). Estilos de aprendizaje en estudiantes de la carrera de odontología de la Universidad Privada Privada Abierta Latinoamericana. Revista Cientifica Multidisciplinaria, 6(4), 111-116.

Calvache-Sánchez, L., Gómez-Ramírez, M. R. \& Poveda-Alonso, O. (2013). Relación entre los estilos de aprendizaje y el uso de las TIC en el proceso de aprendizaje de los estudiantes universitarios. Silogismo, 11(1), 21-43.

Camacho, J. A., Chiappe-Laverde, A. \& López de Mesa, C. (2012). Blended learning y estilos de aprendizaje en estudiantes universitarios del área de la salud. Revista de Educación Médica Superior, 26(1), 27-44.

Cantilo, C., Roura, M. \& Sánchez, A. (2012). Tendencias actuales en el uso de dispositivos móviles en educación. La Educación, 147. Recuperado de http://educoas.org/portal/la_educacion_ digital/147/pdf/ART_UNNED_EN.pdf

Castejón, C. \& Pérez, S. (1998). Un modelo causal-explicativo sobre la influencia de las variables psicosociales en el rendimiento académico. Revista Bordon, 2(50), 170-184.

Chen-Kang, L. \& Manjit-Singh, S. (2015). Engineering students learning preferences in UNITEN: Comparative study and patterns of learning styles. Journal of Educational Technology \& Society, 18(3), 266-281.

Cohen, J. (1977). Statistical power analysis for the behavioral science. New York: Academic Press.

Colorado-Aguilar, B. L. \& Edel-Navarro, R. (2012). La usabilidad TIC en la práctica educativa. Revista de Educación a Distancia, 30(2). Recuperado de http://www.um.es/ead/red/30/edel.pdf

Cuña, I. D., Gutiérrez, M., Barón, F. J. \& Labajos, M. T. (2014). Influencia del nivel educativo de los padres en el rendimiento académico, las estrategias de aprendizaje y los estilos de aprendizaje, desde la perspectiva de género. Revista de Estilos de Aprendizaje, 7(13), 64-84. 
Curry, L. (1983). An organization of learning style theory and constructs. In L. Curry (Ed.), Learning style in continuing medical education (pp. 115-131). Ottawa: Canadian Medical Association.

De Miguel, M. \& Arias, J. M. (1999). La evaluación del rendimiento inmediato en la enseñanza universitaria. Revista de Educación, 320, 353-377.

Di Gresia, L., Porto, A., Ripani, L. \& Sosa-Escudero, W. (2003). Rendimiento de los estudiantes de las universidades públicas argentinas. Trabajo presentado en la $36^{\circ}$ Jornadas Internacionales de Finanzas Públicas, Universidad Nacional de Córdoba.

Donche, V., De Maeyer, S., Coertjens, L., Van Daal, T. \& Van Petegem, P. (2013). Differential use of learning strategies in first year higher education: The impact of personality, academic motivation, and teaching strategies. British Journal of Educational Psychology, 83(2), 238-251. https://doi.org/10.1111/bjep.12016

Eco, U. (2010). Umberto Eco: En el futuro, la educación deberá enseñar a comparar sitios web. Recuperado de http://www.revistaenie. clarin.com/literatura/educacion-deberaensenar-comparar-Web_ 0_352764926.html

Escurra-Mayaute, L. M. (2006). Análisis psicométrico del Inventario de Estrategias de Aprendizaje y Estudio en estudiantes universitarios de psicología de Lima metropolitana. Persona, 9, 127-170.

Fernández-Zalazar, D. \& Neri, C. (2013). Psicología educacional y orientación vocacional. Anuario de Investigaciones, 20(1), 153-158.

Flores-Mejía, J. G., Velázquez-Gatica, B. \& Gaona-Rojas, F. (2014). Actitudes, estrategias y estilos de aprendizaje en estudiantes universitarios de Psicología. XI Coloquio Internacional Multidisciplinario. Unidad Profesional del Balsas de la Universidad Michoacana de San Nicolás de Hidalgo (pp. 1-14). Huetamo, Michoacán. Recuperado de http:// www.guillermofloresuagro.net/uploads/5/8/4/5/58453193/ actitudes_estrategias_y_estilos_de_aprendizaje_en_estudiantes_universitarios_de_psicologi\%CC\%81a.pdf 
Freiberg-Hoffmann, A. \& Fernández-Liporace, M. M. (2013). Cuestionario Honey-Alonso de Estilos de Aprendizaje: Análisis de sus propiedades psicométricas en estudiantes universitarios. Revista Summa Psicológica UST, 10(1), 103-117.

Freiberg-Hoffmann, A., Ledesma, R. \& Fernández-Liporace, M. M. (2017, en prensa). Análisis de las propiedades psicométricas del Inventario de Estrategias de Aprendizaje y Estudio (LASSI) en Estudiantes Universitarios. Revista Iberoamericana de Diagnóstico y Evaluación Psicológica, 44(2).

Garbanzo-Vargas, G. M. (2007). Factores asociados al rendimiento académico en estudiantes universitarios, una reflexión desde la calidad de la educación superior pública. Educación, 31(1), 43-63. https://doi.org/10.15517/revedu.v31i1.1252

Gargallo, B., Almerich, G., Suárez-Rodríguez, J. \& García-Félix, E. (2012). Estrategias de aprendizaje en estudiantes universitarios excelentes y medios. Su evolución a lo largo del primer año de carrera. Revista Electrónica de Investigación y Evaluación Educativa, 18(2). https://doi.org/10.7203/relieve.18.2.2000

Gravini, M. L. \& Iriarte, F. (2008). Procesos metacognitivos de estudiantes con diferentes estilos de aprendizaje. Psicología desde el Caribe, 22, 1-24. Recuperado de http://www. scielo.org.co/scielo.php?script=sci_arttext\&pid=S0123417X2008000200002\&lang=pt

Griffin, R., MacKewn, A., Mosare, E. \& VanVuren, K. W. (2012). Do learning and study skills affect academic performance? An empirical investigation. Contemporary Issues in Education Research, 5(2), 109-116. https://doi.org/10.19030/cier. v5i2.6928

Hallin, K. (2014). Nursing students at a university - A study about learning style preferences. Nurse Education Today, 34(12), 14431449. https://doi.org/10.1016/j.nedt.2014.04.001

Heck, R. H., Thomas, S. L. \& Tabata, L. N. (2011). Multilevel and longitudinal modeling with IBM SPSS. New York: Routledge. 
Honey, P. \& Mumford, A. (1986). The manual of learning styles. Maidenhead, Berkshire: P. Honey, Ardingly House.

Ibarra, M. C. \& Michalus, J. C. (2010). Análisis del rendimiento académico mediante un modelo logit. Ingeniería Industrial, 9(2), 47-56.

Jiraporncharoen, W., Angkurawaranon, C., Chockjamsai, M., Deesomchok, A. \& Euathrongchit, J. (2015). Learning styles and academic achievement among undergraduate medical students in Thailand. Journal of Educational Evaluation for Health Professions, 12(38). https://doi.org/10.3352/jeehp.2015.12.38

Jouhari, Z., Haghani, F. \& Changiz, T. (2016). Assessment of medical students' learning and study strategies in self-regulated learning. Journal of Advances in Medical Education \& Professionalism, 4(2), 72-79.

Keefe, J. W. (1988). Profiling and utilizing learning style. Reston, Virginia: NASSP.

Kolb, A. \& Kolb, D. (2005). The Kolb learning style inventory - version 3.12005 technical specifications. HayGroup. Recuperado de www.whitewater-rescue.com/support/pagepics/lsitechmanual. pdf

Ku, D. \& Chang, C. (2011). The effect of academic discipline and gender difference on taiwanese college students' learning styles and strategies in web based learning environments. The Turkish Online Journal of Educational Technology, 10(3), 265-272.

Lee, J., Sax, L., Kim, K. \& Hagedom, L. (2004). Understanding students' parental education beyond first generation status. Community College Review Summer, 32(1), 1-20. https://doi. org/10.1177/009155210403200101

López-Aguado, M. (2011). Estilos de aprendizaje. Diferencias por género, curso y titulación. Revista Estilos de Aprendizaje, 7(7), 109-134.

Loomis, K. (2000). Learning styles and asynchronous learning: Comparing the LASSI model to class performance. Journal of Asynchronous Learning Networks, 4(1), 23-32. 
Loret de Mora Garay, J. E. (2011). Estilos y estrategias de aprendizaje en el rendimiento académico de los estudiantes de la universidad peruana Los Andes de Huancayo Perú. Revista de Estilos de Aprendizaje, 8(8), 149-184.

Marchesi, A. (2000). Un sistema de indicadores de desigualdad educativa. Revista Iberoamericana de Educación, 23, 1-22.

Marland, J., Dearlove, J. \& Carpenter, J. (2015). LASSI: An Australian evaluation of an enduring study skills assessment tool. Journal of Academic Language \& Learning, 9(2), 32-45.

Martín-García, A. V. \& Rodríguez-Conde, M. J. (2003). Estilos de aprendizaje y grupos de edad: comparación de dos muestras de estudiantes jóvenes y mayores. Aula Abierta, 82, 97-114.

Martínez-Garrido, C. \& Murillo, F. (2014). Programas para la realización de modelos multinivel. Un análisis comparativo entre MLwiN, HLM, SPSS y Stata. Revista Electrónica de Metodología Aplicada, 19(2), 1-24.

Maureira, F., Durán, F., Pastén, S., Herrera, M. P., Urquejo, P. \& Opazo, L. (2015). Independencia de los estilos de aprendizaje de Kolb y las inteligencias múltiples en estudiantes de Educación Física de la USEK de Chile. Psiquiatría Universitaria, 11(2), 209-215.

Mehrdad, A. G. \& Ahghar, M. (2012). Learning styles and learning strategies of left-handed EFL students. Procedia-Social and Behavioral Sciences, 31, 536-545. https://doi.org/10.1016/j. sbspro.2011.12.100

Meso, K., Pérez, J. \& Mendiguren, T. (2011). La implementación de las redes sociales en la enseńanza superior universitaria. Tejuelo, 12, 137-155.

Ministerio de Hacienda, Buenos Aires Ciudad (abril de 2016). Población estudiantil de instituciones universitarias de la Ciudad de Buenos Aires. Año 2012. Recuperado de https://www.estadisticaciudad. gob.ar/eyc/wp-content/uploads/2016/04/ir_2016_998.pdf

Ministerio de Educación de la Nación (mayo de 2016). Estructura del nivel de educación secundaria. Recuperado de http://portal. educacion.gov.ar/secundaria/estructura-del-nivel/ 
Miles, J. \& Banyard, P. (2007). Understanding and using statistics in psychology. California: SAGE Publications, Inc.

Morales, A. (1999). El entorno familiar y el rendimiento escolar. Andalucía: Consejería de Educación y Ciencia.

Morán, L. (2012). Blended learning. Desafío y oportunidad para la educación actual. Revista Electrónica de Tecnología Educativa, 39. Recuperado de http://edutec.rediris.es/Revelec2/Revelec39/ blended_learning_desafio_oportunidad_educacion_actual.html

Moya-Martínez, M. V., Hernández-Bravo, J. R., Hernández-Bravo, J. A. \& Cózar-Gutiérrez, R. (2011). Análisis de los estilos de aprendizaje y las TIC en la formación personal del alumno universitario a través del cuestionario REATIC. Revista de Investigación Educativa, 29(1), 137-156.

Muelas, A. \& Navarro, E. (2015). Learning strategies and academic achievement. Procedia-Social and Behavioral Sciences, 165, 217-221. https://doi.org/10.1016/j.sbspro.2014.12.625

Muñetón, M. J., Pinzón, M. A., Alarcón, L. L. \& Olaya, C. I. (2013). Learning styles and strategies related to academic achievement among university students. Pensamiento Psicológico, 11(1), 115-129.

Navarro-Jiménez, M. J. (2008). Como diagnosticar y mejorar los estilos de aprendizaje. Almería, España: Asociación Procompal.

Ortiz-Fernández, L., Moromi-Nakata, H., Quintana del Solar, C., Barra-Hinostroza, M., Bustos de la Cruz, J., Cáceres, L., CheinVillacampana, S. \& Rodríguez-Vargas, C. (2014). Estrategias, estilos de aprendizaje y rendimiento académico en estudiantes ingresantes de Odontología. Odontología Sanmarquina, 17(2), 76-81. https://doi.org/10.15381/os.v17i2.11050

Ortiz-Ojeda, A. F. \& Canto-Herrera, P. J. (2013). Estilos de aprendizaje y rendimiento académico en estudiantes de Ingeniería en México. Revista de Estilos de Aprendizaje, 11(11), 160-177.

Padilla-Miranda, A. M., Gutiérrez-Fonseca, E. \& Cabrera-Campos, A. (2013). Aproximación a los estilos de aprendizaje de estudiantes 
Estilos y estrategias de aprendizaje en estudiantes universitarios de Buenos Aires / Freiberg et al.

de la Universidad de las Ciencias Informáticas de la Habana, Cuba. Revista de Estilos de Aprendizaje, 11(11), 190-211.

Pardo, A., Ruiz, M. \& San Martín, R. (2007). Cómo ajustar e interpretar modelos multinivel con SPSS. Psicothema, 19(2), 308-321.

Peixoto, H. M., Peixoto, M. M. \& Alves, E. D. (2012). Learning strategies used by undergraduate and postgraduate students in hybrid courses in the area of health. Revista Latino-Americana de Enfermagem, 20(3), 551-558. https://doi.org/10.1590/S010411692012000300017

Pintos, M.E. (2012, 26 de marzo). En el primer año, el 58\% de los estudiantes dejan o cambian de carrera. Clarín.com, Sociedad, Recuperado de http://www.clarin.com/sociedad/primer-estudiantes-dejan-cambian-carrera_0_670732977.html

Ponce de León Castañeda, M. E., Varela-Ruiz, M., Lozano-Sánchez, J. R., Ortiz-Montalvo, A. \& Ponce-Rosas, R. E. (2010). Perfil de preferencias de aprendizaje de alumnos y profesores de medicina. Elemento a considerar en la enseñanza. Educación Médica, 13(1), 33-39. https://doi.org/10.4321/S1575-18132010000100007

Porcel, E., Dapozo, G. \& López, M. (2010). Predicción del rendimiento académico de alumnos del primer año de la FACENA (UNNE) en función de su caracterización socioeducativa. Revista Electrónica de Investigación Educativa, 12(2), Recuperado de http://redie.uabc.mx/redie/article/view/264/730

Porto, A. \& Di Gresia, L. (abril, 2001). Rendimiento de estudiantes universitarios y sus determinantes. Anales de la Asociación Argentina de Economía Política, Buenos Aires. Recuperado de http:// www.aaep.org.ar/anales/works/works2001/porto_a.pdf

Pujol, L. (2008). Búsqueda de información en hipermedios: efecto del estilo de aprendizaje y el uso de estrategias metacognitivas. Investigación y Postgrado, 23(3), 45-67.

Ross, L. R. \& Powell, R. (1999). Relationships between gender and success in distance education courses: a preliminary investigation. Research in Distance Education, 2(2), 10-11. 
Sánchez-Socarrás, V., Donat-Roca, R., Fornons-Fontdevilla, D. \& Vaqué-Crusellas, C. (2015). Análisis del comportamiento de los estilos de aprendizaje en estudiantes universitarios y profesionales de ciencias de la salud. Revista de Estilos de Aprendizaje, 8(16), 137-161.

Simsec, A. \& Balaban, J. (2010). Learning strategies of successful university students. Contemporary Educational Technology, 1(1), 36-45.

Sora, B., Caballer, A. \& Peiró, J. (2011). Consecuencias de la inseguridad laboral. El papel modulador del apoyo organizacional desde una perspectiva multinivel. Psicothema, 23(3), 394-400.

Stover, J. B., Uriel, F. \& Fernández-Liporace, M. M. (2012). Inventario de estrategias de Aprendizaje y Estudio: Análisis psicométricos de una versión abreviada. Revista Argentina de Ciencias del Comportamiento, 4(3), 4-12.

Stroup, W. (2013). Generalized linear mixer models. New York: Taylor $\&$ Francis Group, LCC.

Tejedor-Tejedor, F. J. (2003). Poder explicativo de algunos determinantes del rendimiento académico en los estudios universitarios. Revista Española de Pedagogía, 224, 5-32.

Valle, A., González, R., Cuevas, L. M. \& Fernández, A. P. (1998). Las estrategias de aprendizaje: características básicas y su relevancia en el contexto escolar. Revista de Psicodidáctica, 6, 53-68.

Ventura, A. C. (2011). Estilos de aprendizaje y prácticas de enseñanza en la universidad. Un binomio que sustenta la calidad educativa. Perfiles Educativos, 33, 142-154.

Ventura, A. C., Moscoloni, N. \& Gagliardi, R. P. (2012). Estudio comparativo sobre los estilos de aprendizaje de estudiantes universitarios argentinos de diferentes disciplinas. Psicología desde el Caribe, 29(2). Recuperado de http://rcientificas.uninorte.edu. co/index.php/psicologia/article/view/3922/3919

Villalba, A. (2015). Estilos de aprendizaje en alumnos universitarios de profesorado en Biología y Licenciatura en Biodiversidad. Revista de Estilos de Aprendizaje, 8(16), 79-100. 
Weinstein, C. \& Mayer, R. E. (1986). The teaching of learning strategies. In Wittrock, M. C. (Ed.), Handbook of research in teaching (3ra. ed.) (pp. 315-327). New York: Macmillan.

Weinstein, C. E. Schulte, A. C. \& Palmer, D. R. (1987). Learning and Study Strategies Inventory (LASSI). Clearwater. F1: H \& H Publishing.

Williams, B., Brown, T. \& Etherington, J. (2013). Learning style preferences of undergraduate pharmacy students. Currents in Pharmacy Teaching and Learning, 5(2), 110-119. https://doi. org/10.1016/j.cptl.2012.09.003

Yip, M. C. (2012). Learning strategies and self efficacy as predictors of academic performance: A preliminary study. Quality and Higher Education, 18(1), 23-34.https://doi.org/10.1080/13538322.20 12.667263

Zapata-Castañeda, P., Oviedo, P., Cárdenas-Salgado, F., RendónFernández, M., Gómez-Hurtado, M., López, M. \& Figueroa, L. (2012). Los estilos de aprendizaje de los estudiantes de la Universidad de La Salle según el modelo de Kolb y sus implicancias para la didáctica universitaria. Actualidades Pedagógicas, $60,123-147$.

Recibido: 7 de junio, 2016 Revisado: 19 de febrero, 2017 Aceptado: 22 de febrero, 2017 